\title{
Gold nanoparticles based digital color analysis for quinidine detection
}

\author{
ZOU BoZhou ${ }^{1}$, LIU Yue $^{1}$, YAN XiaoLi ${ }^{2} \&$ HUANG ChengZhi ${ }^{1,2 *}$ \\ ${ }^{1}$ College of Chemistry and Chemical Engineering, Education Ministry Key Laboratory on Luminescence and Real-Time Analysis, Southwest \\ University, Chongqing 400715, China; \\ ${ }^{2}$ College of Pharmaceutical Sciences, Southwest University, Chongqing 400715, China
}

Received October 19, 2012; accepted December 14, 2012; published online April 9, 2013

\begin{abstract}
In this contribution, we developed a novel digital color analysis of gold nanoparticles (AuNPs) for pharmaceutical detection by choosing quinidine as an example. It was found that the color of AuNPs colloid solution changed from red to blue in the presence of different concentrations of quinidine, and the color information of each solution could be digitalized with tricolor (RGB) system. Under the optimum conditions, the $R$ value of the solution was found to be linear with the natural logarithm of concentration of quinidine in the range of $112-384 \mathrm{nmol} / \mathrm{L}$. This method has the advantages of rapid, economical and simple, and can serve as a potential alternative to traditional spectrophotometry for practical use.
\end{abstract}

digital color analysis, gold nanoparticles, colorimetric, quinidine

Citation: $\quad$ Zou B Z, Liu Y, Yan X L, et al. Gold nanoparticles based digital color analysis for quinidine detection. Chin Sci Bull, 2013, 58: 2027-2032, doi: 10.1007/s11434-013-5708-3

Recently, digital color analysis has attracted considerable attentions because of its high sensitivity, facility and rapidity, wherein the color is measured and transformed into numerical values that can be treated as signals for analysis through color system (RGB or HSB system). To achieve the goal of analysis, it only needs a digital color recorder (CCD or digital camera) to obtain color images and a software to code and read out color information [1]. The use of digital images provides a current opportunity to develop convenient, practical and quantitative method for analytical chemistry. For instance, digital color analysis has been successfully applied to the sensing of metal ions [1-3], small molecules [4,5], gas [6,7] and bacteria [8], and also used to estimate the particle size, morphology and composition of metal nanomaterials [9], monitor refractive index of surrounding medium and study the interaction between plasmonic particles and molecules adsorbed on them [10,11].

Metal nanoparticles based colorimetric assays have been widely explored for analytical purpose during the past dec-

*Corresponding author (email: chengzhi@swu.edu.cn) ade [12]. Because of their strong localized surface plasmon resonance, the colloid solution of metal nanoparticles appears intense color. In particular, the aggregated nanoparticles solution shows a different color from the well dispersed one, which forms the basis of the colorimetric analysis [13-15]. This vivid color change induced by aggregation or dispersion can be observed by naked eye, and thus it does not require any sophisticated instruments. Although the naked-eye based colorimetric detection is simple, rapid and sensitive, it cannot serve as a quantitative method without the assistance of UV-Vis spectrophotometer.

To overcome this shortcoming, herein we introduce digital color analysis system to characterize the color change of AuNPs solution induced by aggregation. It is known that the color of AuNPs colloidal solution is red, and it was found that quinidine could induce the aggregation of FSN-AuNPs, leading to the color of colloidal solution change from red to blue. By utilizing RGB color system to analyze the color information of colloidal solution, we can determine the concentration of quinidine without any complicated instruments. Therefore, digital color analysis based on gold nanoparticles 
(AuNPs) could provide a cheap, portable and sensitive analytical method, which can be extended for practical use.

\section{Experimental}

\subsection{Materials and apparatus}

Chloroauric acid tetrahydate $\left(\mathrm{HAuCl}_{4} \cdot 4 \mathrm{H}_{2} \mathrm{O}\right)$ was obtained from Sinopharm Chemical Reagent Co., Ltd (Shanghai, China). Zonyl ${ }^{\circledR}$ FSN-100 and quinidine were purchased from Sigma-Aldrich. Other chemicals were analytical reagent grade for use without further purification. $50 \mathrm{mmol} / \mathrm{L}$ MES buffer ( $\mathrm{pH}$ 6.5) was employed to adjust the acidity of the aqueous solutions. Milli-Q purified water (18.2 M $\Omega$ ) was used for all sample preparations.

The absorption spectra of AuNPs colloid solution was measured with U-3010 UV-Vis spectrophotometer (Hitachi, Japan). Scanning electron microscopy (SEM) images were obtained using S-4800 Scanning Electron Microscope (Hitachi). Dark-field light scattering (DFLS) imaging was carried out through BX51 Optical Microscope (Olympus, Japan). Zetasizer Nano ZS (Malvern, America) was used to measure the zeta potential. Photographs were taken with E-510 digital camera (Olympus). A QL-901 vortex mixer (Haimen, China) was employed to mix the solutions. HH-4 numerical show constant temperature tank (Ronghua, China) was used to control the temperature of solutions. The images were analyzed using Image-Pro Plus (IPP) software (Media Cybernetics, America).

\subsection{Synthesis of AuNPs and modification with FSN-100}

AuNPs with an average diameter of $13 \mathrm{~nm}$ were prepared following the classical method by reducing $\mathrm{HAuCl}_{4}$ with citrate in aqueous solution [16]. The $52 \mathrm{~mL}$ mixture containing $50 \mathrm{~mL}$ water and $2 \mathrm{~mL} \mathrm{HAuCl}_{4}$ solution (w/v, $1 \%$ ) was boiled under stirring, and $1 \mathrm{~mL}$ of trisodium citrate solution $(\mathrm{w} / \mathrm{v}, 5 \%)$ was added into the above solution under vigorous stirrig. After boiling for $10 \mathrm{~min}$ and further stirring for $15 \mathrm{~min}$, the solution was allowed to cool down to room temperature with continued stirring, and then $1 \mathrm{~mL}$ of nonionic fluorocarbon surfactant FSN-100 solution (0.075 $\mathrm{g} / \mathrm{mL}$ ) was added. The colloid solution was stored at $4^{\circ} \mathrm{C}$ for further use. The concentrations of AuNPs was measured by Lambert- beer's law $(A=\varepsilon b c)$ to be $6 \mathrm{nmol} / \mathrm{L}$.

\subsection{Detection of quinidine}

The $0.1 \mathrm{~mL}$ of $50 \mathrm{mmol} / \mathrm{L}$ MES buffer and an appropriate volume of the quinidine solution $\left(5 \times 10^{-3} \mathrm{~g} / \mathrm{L}\right)$ were added to a $1.5 \mathrm{~mL}$ glass bottle, and then the mixture was diluted to $0.8 \mathrm{~mL}$ with ultra-pure water and mixed thoroughly. Next, $0.2 \mathrm{~mL}$ of AuNPs colloid solution was added, and then the mixture was vortexed and incubated in a water bath at $65^{\circ} \mathrm{C}$ for $30 \mathrm{~min}$. After that, the solution was photographed by
E-510 digital camera and transferred for absorption spectra measurement by U-3010 UV-Vis spectrophotometer. To avoid the interference of natural light, photographs were taken in a fixed position of a closed room which equipped with a fluorescent lamp (Osram, L36W/765) as light source.

\section{Results and discussion}

\subsection{Spectral features and digital color analysis}

In the oscillating electromagnetic field of the light, the free electrons of AuNPs undergo a collective coherent oscillation, which is termed as localized surface plasmon resonance (LSPR) oscillation. This process is resonant at a particular frequency of the incident light, leading to the absorption and scattering of selective wavelengths of lights [17]. For example, our prepared FSN coated AuNPs have characterized LSPR absorption band at $520 \mathrm{~nm}$, while that of 50 $\mathrm{nm} \mathrm{Ag} \mathrm{nanoparticles} \mathrm{is} \mathrm{characterized} \mathrm{at} \mathrm{about} 410 \mathrm{~nm}$ [18]. LSPR absorption of AuNPs, as reported, not only depends on its size and shape, but also on its surrounding surface environment (e.g. solvent, ligand, and inter-nanoparticle spacing) $[19,20]$. Owing to the reduction of the internanoparticle spacing induced by the aggregation of AuNPs, the strong plasmon coupling between neighboring nanoparticles consequently influences the LSPR absorption [21]. As Figure 1(a) shows, the original absorbance at $520 \mathrm{~nm}$ decreased upon the addition of quinidine, generating a new absorption band centered at $650 \mathrm{~nm}$, which increased as the concentration of quinidine increased. This phenomenon indicated that the aggregation of AuNPs has been triggered directly by quinidine, which could be estimated by the absorbance ratio between 650 and $520 \mathrm{~nm}\left(A_{650} / A_{520}\right)$ as discussed later.

It is known that the LSPR absorption of AuNPs is satisfied at visible wavelengths, therefore attributing for the intense color of colloidal solution [22]. The prepared colloidal AuNPs have a wine red color in the absence of quinidine (inset in Figure 1(a)). This wine red color, however, changed to purple upon the addition of quinidine, and then to blue with increasing the concentration of quinidine in the range of 0-640 nmol/L. In such case, the color change could be characterized with digital color analysis system. As we know, the RGB color system is the most accepted color system and have been widely used to characterize the color, wherein any color can be reproduced by mixing red (R), green $(\mathrm{G})$, and blue (B) color in various ways. In this system, a color can be represented by a group of integer values of $R, G$, and $B$ ranging from 0 to 255 . For example, RGB values of (255 00 ) represents red and (0 2550$)$ refers to green [4]. To extend the analytical use of the color of colloidal gold, we transformed the color information of colloidal solutions into numerical values through the RGB color system. First, the solutions were taken photograph with a digital camera. Next, a selected area of each solution (each 

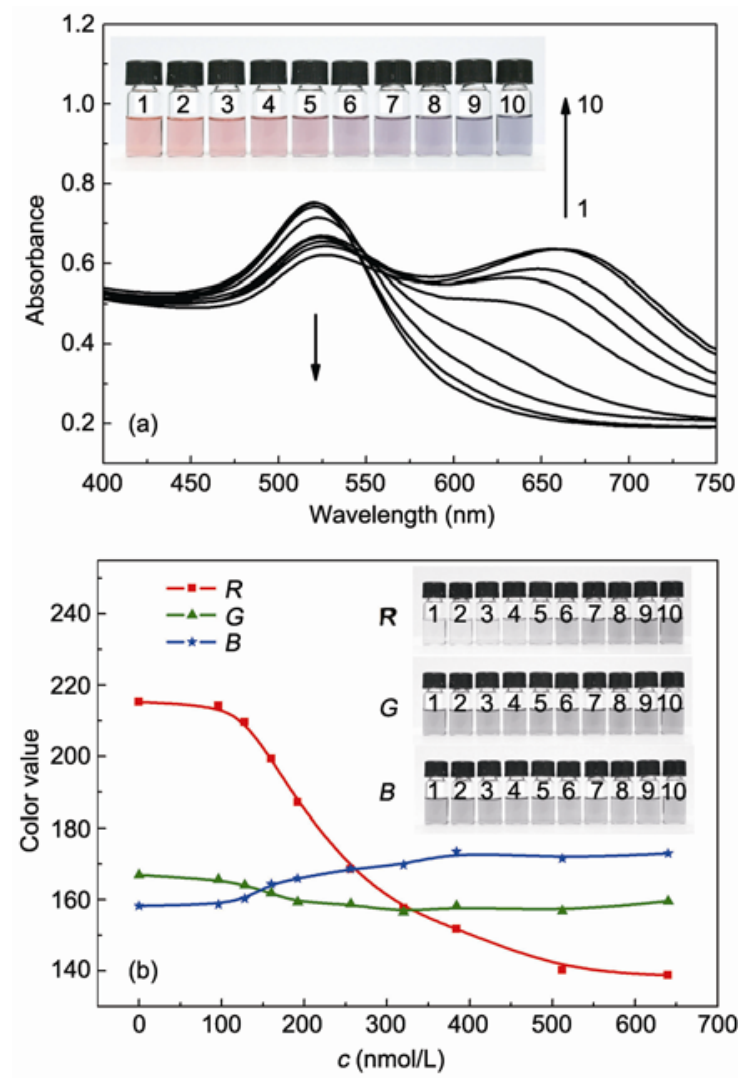

Figure 1 Digital color analysis of AuNPs upon the addition of quinidine. (a) UV-Vis absorption spectra of AuNPs upon the addition of different concentrations of quinidine. Inset showed the corresponding color images of gold colloidal solution. (b) The $R, G, B$ values of colloidal gold changed upon addition of different concentrations of quinidine. Inset showed images of each color channel. Concentrations: quinidine (in the order of 1-10): $0,96,128,160,192,256,320,384,512$ and $640 \mathrm{nmol} / \mathrm{L}$.

area has the same number of pixels) in the photograph was analyzed, as the $R, G, B$ values of the solution color were read out by IPP software in a personal computer. In our study, we found that the values of $R$ channel of the solution obviously decreased with increasing the concentration of quinidine, while the values of $G$ and $B$ channels changed slightly (Figure 1(b)). Thus, we focused on the changes of $R$ values in the following experiments.

The quinidine-triggered aggregation of AuNPs is further confirmed by SEM and DFLS imaging (Figure 2). In the absence of quinidine, AuNPs were uniform disperse particles (Figure 2(a)), which had weak scattering light to be observed under dark-field microscope (Figure 2(d)). While in the presence of $6.4 \mu \mathrm{mol} / \mathrm{L}$ quinidine, AuNPs aggregated mildly (Figure 2(b)). Correspondingly, more scattering light spots were observed (Figure 2(e)) due to plasmon coupling of AuNPs, which led to strong enhanced scattering intensity. When the quinidine concentrations increased to 12.8 $\mu \mathrm{mol} / \mathrm{L}$, obvious aggregation of the AuNPs occurred with the size of the aggregates ranging from hundreds nanometers to micrometers (Figure 2(c)), making the possibility of observing the strong scattering light spots (Figure 2(f)).
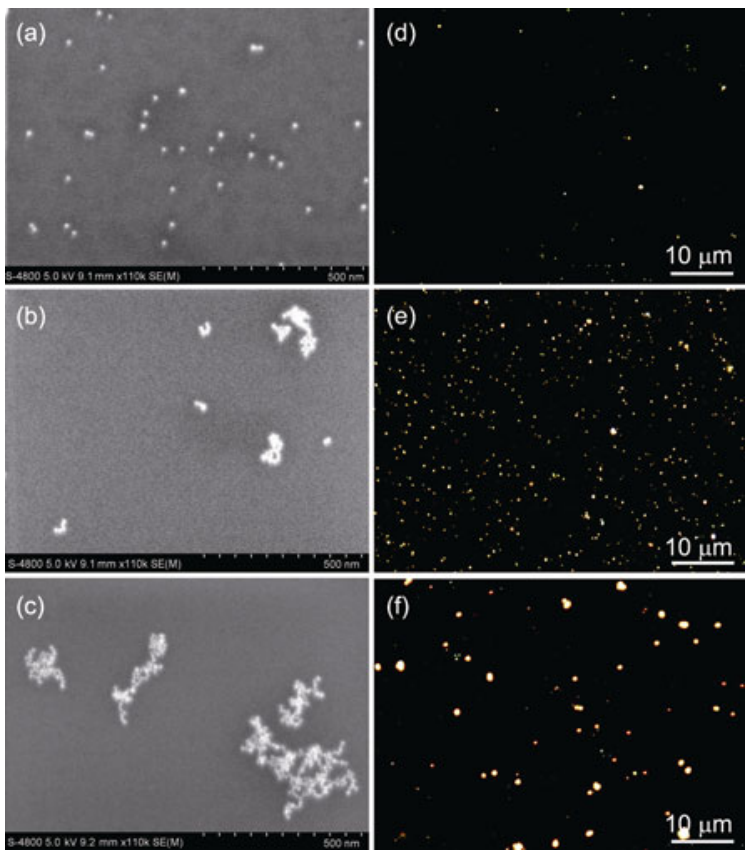

Figure 2 Aggregation of AuNPs as showed by SEM images (a)-(c) and DFLS images (d-f) in the absence of quinidine ((a),(d)) and presence of 6.4 $\mu \mathrm{mol} / \mathrm{L}((\mathrm{b}),(\mathrm{e}))$ and $12.8 \mu \mathrm{mol} / \mathrm{L}((\mathrm{c}),(\mathrm{f}))$ quinidine, respectively.

These observations clearly indicated that quinidine can directly induce the aggregation of FSN modified AuNPs.

\subsection{Mechanism of aggregation of AuNPs}

Quinidine is a kind of pharmaceutical alkaloid that acts as an antiarrhythmic agent in the heart. It contains a endocyclic $\mathrm{N}$ and a tertiary $\mathrm{N}$ atoms (inset of Figure 3 ) with $\mathrm{p} K_{\mathrm{a}}$ values of 4.2 and 8.6 [23], respectively. As previously reported, quinidine acts in its cationic form with these $\mathrm{N}$ atoms predominantly protonated at physiological $\mathrm{pH}$ [24]. Therefore we presume that quinidine is positively charged under our designed experimental conditions ( $\mathrm{pH}$ 6.5), and could electrostatically bind to the negatively charged AuNPs. This is further confirmed by zeta-potential measurement, as shown in Figure 3. The negative charge of the FSN-AuNPs decreases gradually as the concentration of quinidine increase, which proves the existence of the electrostatic binding between them. In addition, quinidine with electron-rich nitrogen atoms is likely to be adsorbed on the surface of AuNPs through the coordinating interaction [15]. As a result, quinidine exhibits strong binding ability to AuNPs and induces crosslinking (aggregation) of AuNPs.

\subsection{Optimal conditions of the assay}

The interaction between AuNPs and quinidine is strongly influenced by the assay conditions such as media $\mathrm{pH}$, solution ionic strength, incubation temperature and time. Different assay conditions were investigated in our studies. First, the effect of $\mathrm{pH}$ was evaluated at $\mathrm{pH}$ range of 


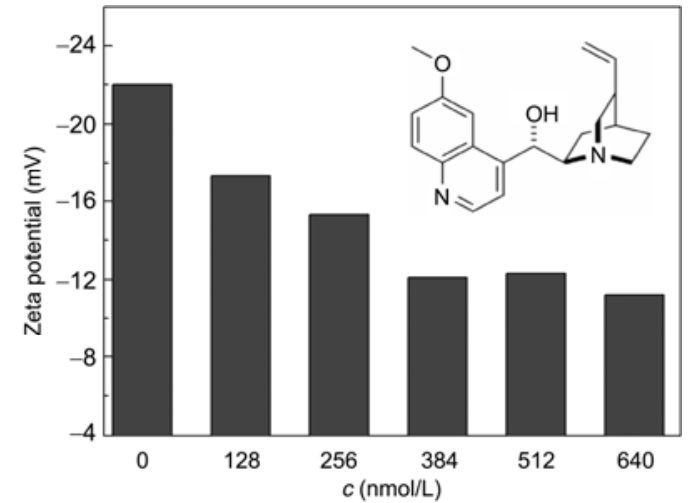

Figure 3 Zeta-potential of AuNPs in the presence of different concentrations of quinidine. Inset showed the molecule structure of quinidine.

3.78-9.62 in BR buffer (Figure S1). At low pH, the dissociation of AuNPs was inhibited, which led to the decrease of negative charge of AuNPs, and thus the electrostatic interaction between AuNPs and positively charged quinidine was weakened. At high $\mathrm{pH}$, however, the protonation of quinidine was inhibited, which reduced the electrostatic interaction as well. Solution ionic strength also has a very important effect on the interaction. When in high ionic strength solution, the electrostatic linkages would be suppressed, leading to a weak crosslinking effect. For these reasons, we chose $5 \mathrm{mmol} / \mathrm{L}$ MES (pH 6.5) as the reaction buffer.

We next explored the effect of temperature on the interaction. It was found that the temperature change in the range of $37-80^{\circ} \mathrm{C}$ influenced the aggregation of AuNPs obviously. The degree of aggregation reached its maximum at $65^{\circ} \mathrm{C}$, as it decreased when the temperature became lower or higher (Figure S2(a)). So $65^{\circ} \mathrm{C}$ was chosen as the operational temperature for all experiments. At this temperature, the reaction was completed after $20 \mathrm{~min}$ and maintained stable within $1 \mathrm{~h}$ (Figure S2(b)).

AuNPs capped with nonionic fluorocarbon surfactant (FSN) molecules were reported to exhibit excellent stability in the presence of high ionic strength solution and a wide range of $\mathrm{pH}$, for FSN could efficiently decrease AuNPs surface tension and prevent them from aggregating [25,26]. In our assay, we used FSN as stabilizer to improve the stability of AuNPs, so it could maintain stable for a long time and avoid the salt induced aggregation of AuNPs under our designed experimental conditions. Besides, it could enhance the response sensitivity to quinidine. The degree of AuNPs aggregation induced by quinidine became larger after capped with FSN (Figure S3), and the $\Delta R$ increased correspondingly.

\subsection{Calibration curve}

The calibration curves were plotted under the optimum conditions. As shown in Figure 4(a), linear relationship was
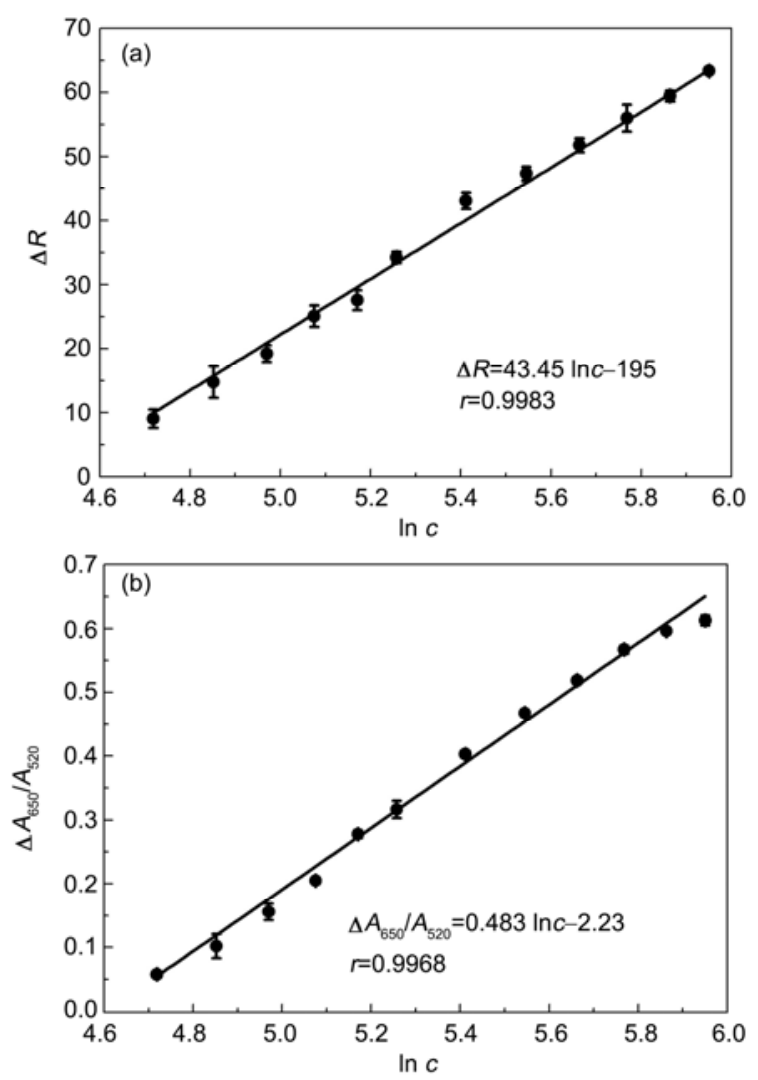

Figure 4 Calibration curve for quinidine under optimum conditions by using (a) digital color analysis and (b) spectrophotometer. The linearity is shown in the figure. All error bars are calculated from three measurements.

obtained between $\Delta R$ and the natural logarithm of concentration of quinidine over the range of 112-384 nmol/L, and the lowest detectable concentration was $96 \mathrm{nmol} / \mathrm{L}$. In order to compare the analytical ability of our proposed method with the spectrophotometry, we measured the absorption of solutions using UV-Vis spectrophotometer at the same time. As shown in Figure 4(b), linear relationship was obtained between $\Delta A_{650} / A_{520}$ and the natural logarithm of concentration of quinidine over the range of $112-384 \mathrm{nmol} / \mathrm{L}$, and the lowest detectable concentration was $96 \mathrm{nmol} / \mathrm{L}$. These results indicated that both methods reveal the similar analytical ability for they had identical linear range and the same lowest detectable concentration.

As reported, the aggregation of AuNPs greatly affects their optical properties even when they form small aggregates, and thus changes the color of colloidal solution [27]. Although the slight change in the optical properties can be detected easily using UV-Vis spectrophotometer, the tiny change in color is hard to be visually observed with naked eye. In such case, the RGB color system can be used to distinguish the tiny difference in colors, which makes our assay have a considerable sensitivity. Compared with some other detections of quinidine [28-31], our proposed method has comparable LOD at $10^{-8} \mathrm{~mol} / \mathrm{L}$ level but with the advantages of portability, time-saving and less cost. 


\section{Conclusions}

In summary, this work describes a new type of simple, commercial and sensitive digital color analysis for quinidine using AuNPs as probes. By transforming color information into numerical values through RGB color system, the AuNPs based colorimetric assay that has been used only as semi-quantitative analysis can serve as an accurate quantitative method. It provides sensitivity and precision comparable to the UV-Vis spectrophotometry, which makes it a potential alternative to traditional visible spectrophotometry. This method is also suitable for colorimetric sensing of any analyte that directly or indirectly induces the AuNPs aggregation or dispersion. In addition, it only needs a digital camera and a PC, thus could be further used in the wild for practical use.

This work was supported by the National Natural Science Foundation of China (21035005) and the National Special Major Project of Science and Technology for Creation of New Drugs (20102X09401-306-1-4).

1 Hirayama E, Sugiyama T, Hisamoto $\mathrm{H}$, et al. Visual and colorimetric lithium ion sensing based on digital color analysis. Anal Chem, 2000, 72: $465-474$

2 Lopez-Molinero A, Linan D, Sipiera D, et al. Chemometric interpretation of digital image colorimetry. Application for titanium determination in plastics. Microchem J, 2010, 96: 380-385

3 Zhan L, Liu Y, Huang C Z. Visual numerical analysis of $\mathrm{Cu}^{2+}$ in typical chinese patent medicines (in Chinese). Sci China Ser B, 2011, 41: 1044-1050

4 Abbaspour A, Khajehzadeh A, Ghaffarinejad A. A simple and cost-effective method, as an appropriate alternative for visible spectrophotometry: Development of a dopamine biosensor. Analyst, 2009, 134: 1692-1698

5 Steiner M S, Meier R J, Duerkop A, et al. Chromogenic sensing of biogenic amines using a chameleon probe and the red-green-blue readout of digital camera images. Anal Chem, 2010, 82: 8402-8405

6 Ali R, Lang T, Saleh S M, et al. Optical sensing scheme for carbon dioxide using a solvatochromic probe. Anal Chem, 2011: 2846-2815

7 Wang X D, Gorris H H, Stolwijk J A, et al. Self-referenced RGB colour imaging of intracellular oxygen. Chem Sci, 2011, 2: 901-906

8 Miranda O R, Li X, Garcia-Gonzalez L, et al. Colorimetric bacteria sensing using a supramolecular enzyme-nanoparticle biosensor. J Am Chem Soc, 2011, 133: 9650-9653

9 Sweeney C M, Nehl C L, Hasan W, et al. Three-channel spectrometer for wide-field imaging of anisotropic plasmonic nanoparticles. J Phys Chem C, 2011, 115: 15933-15937

10 Liu Y, Ling J, Huang C Z. Individually color-coded plasmonic nanoparticles for RGB analysis. Chem Commun, 2011, 47: 8121-8123

11 Jing C, Gu Z, Ying Y L, et al. Chrominance to dimension: A real-time method for measuring the size of single gold nanoparticles. Anal Chem, 2012, 84: 4284-4291

12 Saha K, Agasti S S, Kim C, et al. Gold nanoparticles in chemical and biological sensing. Chem Rev, 2012, 112: 2739-2779

13 Sang Y, Zhang L, Li Y F, et al. A visual detection of hydrogen peroxide on the basis of fenton reaction with gold nanoparticles. Anal Chim Acta, 2010, 659: 224-228

14 Chi H, Liu B, Guan G, et al. A simple, reliable and sensitive colorimetric visualization of melamine in milk by unmodified gold nanoparticles. Analyst, 2010, 135: 1070-1075

15 Li F, Feng Y, Zhao C, et al. Simple colorimetric sensing of trace bleomycin using unmodified gold nanoparticles. Biosens Bioelectron, 2011, 26: 4628-4631

16 Grabar K C, Freeman R G, Hommer M B, et al. Preparation and characterization of au colloid monolayers. Anal Chem, 1995, 67: 735-743

17 Link S, El-Sayed M A. Optical properties and ultrafast dynamics of metallic nanocrystals. Annu Rev Phys Chem, 2003, 54: 331-366

18 Jess W. Optical absorption properties of dispersed gold and silver alloy nanoparticles. J Phys Chem B, 2009, 113: 2647-2656

19 Kelly K L, Coronado E, Zhao L L, et al. The optical properties of metal nanoparticles: The influence of size, shape, and dielectric environment. J Phys Chem B, 2003, 107: 668-677

20 Xu X H N, Huang S, Brownlow W, et al. Size and temperature dependence of surface plasmon absorption of gold nanoparticles induced by tris(2,2'-bipyridine)ruthenium(ii). J Phys Chem B, 2004, 108: $15543-15551$

21 Halas N J, Lal S, Chang W S, et al. Plasmons in strongly coupled metallic nanostructures. Chem Rev, 2011, 111: 3913-3961

22 Jain P K, Huang X, El-Sayed I H, et al. Noble metals on the nanoscale: Optical and photothermal properties and some applications in imaging, sensing, biology, and medicine. Accounts Chem Res, 2008, 41: 1578-1586

23 Vargas R C, Tenreiro S, Teixeira M C, et al. Saccharomyces cerevisiae multidrug transporter Qdr2p (Yil121wp): Localization and function as a quinidine resistance determinant. Antimicrob Agents Chemother, 2004, 48: 2531-2537

24 Yeola S W, Rich T C, Uebele V N, et al. Molecular analysis of a binding site for quinidine in a human cardiac delayed rectifier $\mathrm{K}^{+}$ channel. Circ Res, 1996, 78: 1105-1114

25 Liu Y, Huang C Z. One-step conjugation chemistry of DNA with highly scattered silver nanoparticles for sandwich detection of DNA. Analyst, 2012, 137: 3434-3436

26 Lu C, Zu Y, Yam V W W. Specific postcolumn detection method for hplc assay of homocysteine based on aggregation of fluorosurfactant-capped gold nanoparticles. Anal Chem, 2007, 79: 666-672

27 Turkevich J, Garton G, Stevenson P C. The color of colloidal gold. J Colloid Sci, 1954, 9 (Suppl): 26-35

28 Nielsen F, Nielsen K K, Brøsen K. Determination of quinidine, dihydroquinidine, (3S)-3-hydroxyquinidine and quinidine $N$-oxide in plasma and urine by high-performance liquid chromatography. $\mathrm{J}$ Chromatogr B, 1994, 660: 103-110

29 Zaugg S, Thormann W. Capillary electrophoretic separation, immunochemical recognition and analysis of the diastereomers quinine and quinidine and two quinidine metabolites in body fluids. J Pharm Biomed Anal, 2001, 24: 785-799

30 Yang C X, Huang C Z. Fluorescent microscopic determination of quinidine sulfate in serum samples with self-ordered ring technique by capillary flow effect. Chin J Anal Chem, 2006, 34: 183-186

31 Zeng Y, Cai L, Wang H, et al. Resonance light scattering study on the interaction between quinidine sulfate and congo red and its analytical application. Luminescence, 2010, 25: 30-35

Open Access This article is distributed under the terms of the Creative Commons Attribution License which permits any use, distribution, and reproduction in any medium, provided the original author(s) and source are credited. 